\title{
Image Enlargement using Fractal
}

\author{
King-Hong Chung, Yik-Hing Fung and Yuk-Hee Chan \\ Centre for Multimedia Signal Processing \\ Department of Electronic and Information Engineering \\ The Hong Kong Polytechnic University, Hong Kong
}

\begin{abstract}
In fractal coding technique, an image is encoded by making use of its self-similarity property. The image can be reconstructed with some well-defined contractive mappings based on this property and hence, in theory, the reconstructed image can be of any desirable size by using an initial image of appropriate size during the decoding process. However, in practice, the enlarged image is always degraded due to the sub-optimal contractive mappings used. In this paper, a fractal-based image enlargement technique is proposed to reduce this problem. This technique can preserve the details in edge regions while maintaining the smoothness in flat regions, which is superior to conventional image enlargement techniques such as bilinear interpolation and cubic convolution.
\end{abstract}

\section{INTRODUCTION}

Image enlargement plays an important role in many fields, ranging from medical imaging to military application or to consumer electronics. To a large extent, it relies on image interpolation. The principle of all interpolation schemes is to determine the parameters of a continuous image representation from a set of discrete points. Simple approaches include the zero order (nearest neighbor) and the first order (bilinear) interpolation [1].. The more advanced schemes are the cubic splines [2] and the cubic convolution interpolation $[3,4]$. The common defect of these approaches is their intrinsic inability to reproduce sharp image details. This property is due to the low pass filtering involved in their operations [5].

Fractal can be used as another approach for image enlargement due to the property that the fraction representation of an image carries the same amount of detail regardless of its scale [6]. However, natural images are not exactly self-similar, various artifacts, such as blocking artifact and lost of details, appear in the enlarged images although the sharpness of the original image can be preserved.

For this reason, we propose a fractal-based enlargement technique in this paper to overcome such a problem by introducing an enhancement layer. This paper is organized as follows. In Section 2, an overview of the conventional fractal image coding and its application in image enlargement are given. Section 3 presents the enhancement layer and its use in the enlargement process. Section 4 shows some simulation results and a brief conclusion is given in Section 5 .

\section{FRACTAL IMAGE CODING}

Fractal image coding technique is based on the mathematical theory of iterated contractive transformations developed by Barnsley $[7,8]$. In this technique, the original image $I_{o r i}$, of size $N \times N$, is first partitioned into a number of non-overlapping regions called range blocks of size $n \times n$ each. For each range block $R_{i}$, where $i=1,2,3 \ldots(N / n)^{2}$, the fractal encoder searches for the best matching domain block, $D_{i}$, in a domain pool based on a criterion that $J=\left\|R_{i}-\hat{R}_{i}\right\|^{2}$ is minimum, where $\hat{R}_{i}$ is a transformed version of $D_{i}$.

The domain pool is defined as a set of the domain blocks. The domain blocks in the domain pool are extracted from $I_{\text {ori }}$ by sliding a window of size $n_{d} \times n_{d}$ across $I_{o r i}$ from left to right and from top to bottom. The size of each domain block should be larger than that of a range block to fulfill the contractive requirement [9]. Usually, we let $n_{d}=2 n$.

The affine transform used to get $\hat{R}_{i}$ is generally given as

$$
\hat{R}_{i}=s_{i} \cdot \psi_{i}\left(S\left(D_{i}\right)\right)+o_{i}
$$

where $s_{i}$ and $o_{i}$ are, respectively, the contrast scalar factor and the luminance shift factor to be defined. Operator $\psi_{i}$ is one of the eight possible isomeric transforms purposed in [9]. Operator $S$ is a spatial contractive transform which maps $D_{i}$ onto a block of size $n \times n$. This is usually implemented by averaging four surrounding pixels.

The optimized contrast scalar factor $s_{i}$ and the luminance shift factor $o_{i}$ are calculated as follows. 


$$
s_{i}=\frac{\frac{1}{K} \sum_{j=1}^{K} r_{j} d_{j}-\left(\frac{1}{K} \sum_{j=1}^{K} r_{j}\right)\left(\frac{1}{K} \sum_{j=1}^{K} d_{j}\right)}{\frac{1}{K} \sum_{j=1}^{K} d_{j}^{2}-\left(\frac{1}{K} \sum_{j=1}^{K} d_{j}\right)^{2}}
$$

and $\quad o_{i}=\frac{1}{K} \sum_{j=1}^{K} r_{j}-s_{i}\left(\frac{1}{K} \sum_{j=1}^{K} d_{j}\right)$

where $r_{j}$ and $d_{j}$ are, respectively, the intensity values of the $j^{\text {th }}$ pixels of the range block and the transformed domain block, and $K$ is the total number of pixels in the range block.

After the best matching domain block is found, the corresponding affine transform parameters including $s_{i}$. $o_{i}, \psi_{i}$ and the position of $D_{i}$ in $I_{\text {ori }}$ are then stored for image decoding. This process is carried out block by block until transformation parameters for all blocks are defined.

At the decoding phase, started with an arbitrary initial image, affine mappings are recursively applied to corresponding blocks of the intermediate mapping result of the image. Note that the domain pool is updated as the content of the mapping result changes at each iteration. Eventually it converges to an image close to the original after a few iterations. Image enlarging can be achieved easily by scaling the initial image to the desirable size at the very beginning and then scaling the size of range blocks and domain blocks with the same scale factor at each iteration.

\section{PROPOSED ALGORITHM}

The major idea behind the proposed algorithm is to find an enhancement layer for decoding to reduce the difference between a range block and its associated transformed domain block.

\subsection{Enhancement Layer}

Let $D_{i}$ be the best matching domain block for a range block $R_{i}$ of size $n \times n$ and the optimized affine transformation parameters for this range block be $s_{i}, o_{i}$ and $\psi_{i}$. The corresponding reconstructed block, say $\hat{R}_{i}$, can then be given as $\hat{R}_{i}=s_{i} \cdot \psi_{i}\left(S\left(D_{i}\right)\right)+o_{i}$. Assume that there exists an ideal domain block for $R_{i}$, say $D_{i}^{*}$, such that the original range block can be obtained with the same affine transformation as follows.

$$
R_{i}=s_{i} \cdot \psi_{i}\left(S\left(D_{i}^{*}\right)\right)+o_{i}
$$

The difference between $\psi_{i}\left(S\left(D_{i}^{*}\right)\right)$ and $\psi_{i}\left(S\left(D_{i}\right)\right)$, say $E_{i}$, is then given as

$$
E_{i}=\frac{\left(R_{i}-o_{i}\right)}{s_{i}}-\psi_{i}\left(S\left(D_{i}\right)\right) .
$$

This difference is a vector of size $n \times n$ and its scaled version will be used as an enhancement layer for image enlargement.

\subsection{Image Enlargement with Enhancement Layer}

Assume that the size of the original image $I_{o r i}$ is now scaled to $z N \times z N$, where $z$ is the scaling factor. The initial image is then of size $z N \times z N$. During enlargement, the corresponding enhancement layer for each scaled range block (of size $z n \times z n$ ) is added in the contractive mapping to compensate for the error between $R_{i}$ and $\hat{R}_{i}$. In particular, at the $k^{\text {th }}$ iteration, the contractive mapping of the $i^{\text {th }}$ range block is given by

$$
R_{i(k)}=s_{i} \cdot\left(\Psi_{i}\left(S\left(D_{i(k)}\right)\right)+E_{i}^{\prime}\right)+o_{i}
$$

where $R_{i(k)}$ is the $k^{\text {th }}$ mapping output of the block, $D_{i(k)}$ is a block in $I_{(k-1)}$, the $(k-1)^{t h}$ mapping output of the image, whose position in $I_{(k-1)}$ corresponds to that of $D_{i}$ in $I_{o r i}$, and $E_{i}^{\prime}$ is the interpolated $E_{i}$. Zero order interpolation is applied to interpolate $E_{i}$ to $E_{i}^{\prime}$.

Fractal coding technique is a block based technique and hence block artifact is unavoidable in the reconstructed image. When this technique is applied to enlarge an image, the block artifact is emphasized and could be highly visible. In the proposed approach, in order to remove the block artifact in the enlarged image, the image is enlarged with two different partition schemes and then their results are averaged to get the final result.

In the first partition scheme, $I_{o r i}$ is partitioned into nonoverlapped blocks of size $n \times n$ as usual. As in the second scheme, $I_{\text {ori }}$ is also partitioned into nonoverlapped blocks of size $n \times n$, but the centers of the blocks lie at the corners of the blocks defined in the first partition scheme. Those range blocks at the boundary of the image are not of size $n \times n$ and hence are not handled.

\section{SIMULATION RESULTS}

Simulation was carried out to evaluate the performance of the proposed algorithm. Seven 256-level testing images of size $512 \times 512$ were used in the simulation. The testing images were first downscaled to $256 \times 256$. Then they were enlarged to their original size with different algorithms. The size of range blocks used in this simulation was $4 \times 4$.

Table 1 shows the PSNR performance of various algorithms. Specifically, PSNR is defined as 


$$
P S N R=10 \log \left(\frac{N \times N \times 255 \times 255}{\sum_{j=1}^{N} \sum_{i=1}^{N}\left(p_{i j}-\hat{p}_{i j}\right)^{2}}\right)
$$

where $p_{i j}$ and $\hat{p}_{i j}$ represent the image pixels of the original image and the enlarged image respectively, and $N \times N$ is the size of the images.

On average, the PSNR of all the enlarged images obtained from the proposed algorithm is $1.9 \mathrm{~dB}$ higher than that of the cubic convolution interpolation [3]. In particular, for image "Peppers", the proposed algorithm produces an output of $34.36 \mathrm{~dB}$ which is much higher than those obtained with the others. The reason for this is that the image "Peppers" contains many flat regions and simple edges and hence there is an amount of selfsimilarity in the image, which is extremely suitable for fractal representation.

Fig. 1 shows the enlarged results of some interpolation algorithms. One can observe that the proposed algorithm preserves the details in the edge regions while the others introduce a certain amount of blur in the enlarged images. As for those complex images like "Mandrill", although the performance of various algorithms is very close in terms of PSNR, the subjective quality of the output produced by the proposed algorithm is much better than that of the others. In Fig. 2, one can see that the proposed algorithm can preserve most of the high frequency content while the others cannot. One can also see that the proposed algorithm can effectively remove the block artifact.

The advantage of using the enhancement layer can be revealed in the simulation. In the high frequency regions like the hair regions in "Mandrill", the fineness of the hair in Fig. $2 \mathrm{f}$ is much higher as compared with Fig. $2 \mathrm{e}$, where no enhancement layer is applied. The difference is obvious even though the result is obtained by averaging two conventional fractal interpolation results as the proposed approach does. The blur introduced by the conventional fractal algorithm[7] is remedied by using the enhancement layer.

\section{CONCLUSIONS}

In this paper, a fractal-based image enlargement technique is proposed. The main idea of this method is to reduce the difference between the range blocks and the transformed outputs of their corresponding domain blocks during fractal coding by introducing an enhancement layer. With the use of the enhancement layer, image details can be preserved in the edge regions without destroying the smoothness of the flat regions. Our simulation results show that the proposed algorithm is able to produce an enlarged image closer to the original image as compared with other conventional algorithms such as bilinear interpolation[1] and cubic convolution[3].

\begin{tabular}{|l|c|c|c|c|c|}
\hline & Ours & $\begin{array}{c}\text { Fractal } \\
{[7]}\end{array}$ & $\begin{array}{c}\text { Han \& } \\
\text { Beak's [5] }\end{array}$ & $\begin{array}{c}\text { Cubic } \\
{[3]}\end{array}$ & $\begin{array}{c}\text { Bilinear } \\
{[1]}\end{array}$ \\
\hline Barbara & 24.602 & 23.679 & 24.206 & 24.214 & 24.213 \\
\hline Boat & 30.618 & 28.201 & 28.391 & 28.399 & 28.174 \\
\hline Goldhill & 30.771 & 28.956 & 29.401 & 29.407 & 29.238 \\
\hline Lena & 33.838 & 31.235 & 30.516 & 30.539 & 30.348 \\
\hline Mandrill & 23.062 & 21.716 & 22.792 & 22.795 & 22.686 \\
\hline Peppers & 34.363 & 32.343 & 30.990 & 30.994 & 30.778 \\
\hline Plane & 32.571 & 29.407 & 29.659 & 29.661 & 29.298 \\
\hline
\end{tabular}

Table 1 Comparison of the PSNR performance of various interpolation algorithms (in $\mathrm{dB}$ )

\section{ACKNOWLEDGEMENT}

The work described in this paper was substantially supported by a grant (A046) from the Center for Multimedia Signal Processing, HKPolyU

\section{REFERENCES}

[1] W. K. Pratt, Digital Image Processing, John Wiley \& Sons, INC., 1991.

[2] H. S. Hou and H.C. Andrews, "Cubic splines for image interpolation and digital filtering", IEEE Trans. Acoust., Speech, Signal Processing, Vol.26, pp.508-517, 1978.

[3] R. G. Keys, "Cubic convolution interpolation for digital image processing", IEEE Trans. Acoustics, Speech, and Signal Processing, Vol.29, pp.1153$1160,1981$.

[4] S. K. Park and R. A. Schowengerdt, "Image reconstruction by parametric cubic convolution", Computer Vision, Graphics, and Image Processing, Vol.23, pp.258-272, 1983.

[5] J. K. Han and S. U. Beak, "Parametric cubic convolution scaler for enlargement and reduction of image", IEEE Trans. Consumer Electronics, Vol.46, pp.247-256, 2000.

[6] S. Welstead, Fractal and Wavelet Image Compression Techniques, SPIE Optical Engineering Press, 1999.

[7] Y. Fisher, Fractal Image Compression - Theory and Applications. New York: Springer-Verlag, 1995.

[8] M.F. Barnsley, Fractals Everywhere, New York: Academic, 1993.

[9] A.E. Jacquin, "Image coding based on a fractal theory of iterated contractive image transformations", IEEE Trans. Image Processing Vol.1, pp.18-30, 1992 


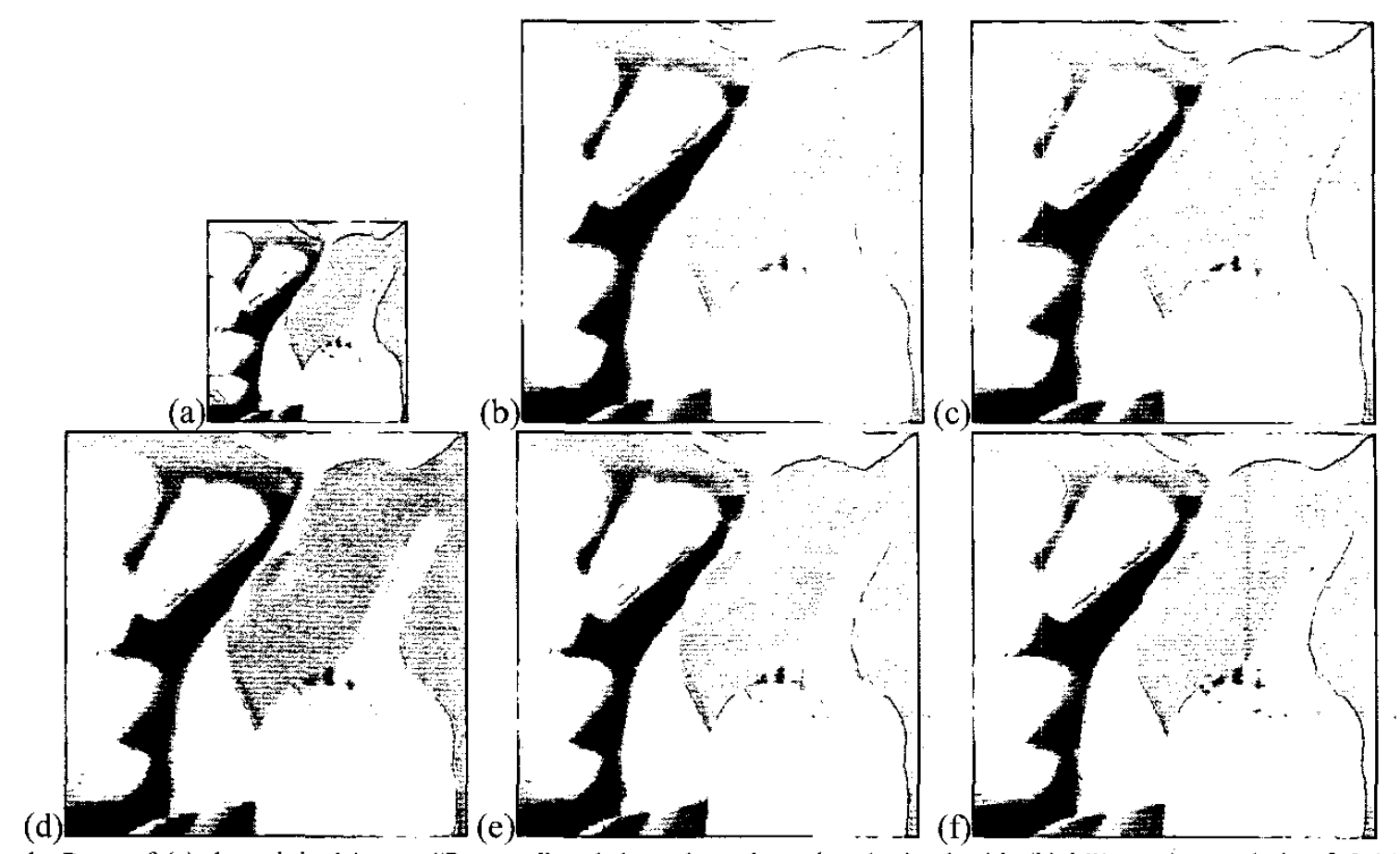

Fig. 1 Parts of (a) the original image "Peppers" and the enlarged results obtained with (b) bilinear interpolation [1] (c) cubic convolution [3], (d) parametric cubic convolution [5], (e) conventional fractal enlargement [7] and (e) the proposed algorithm.

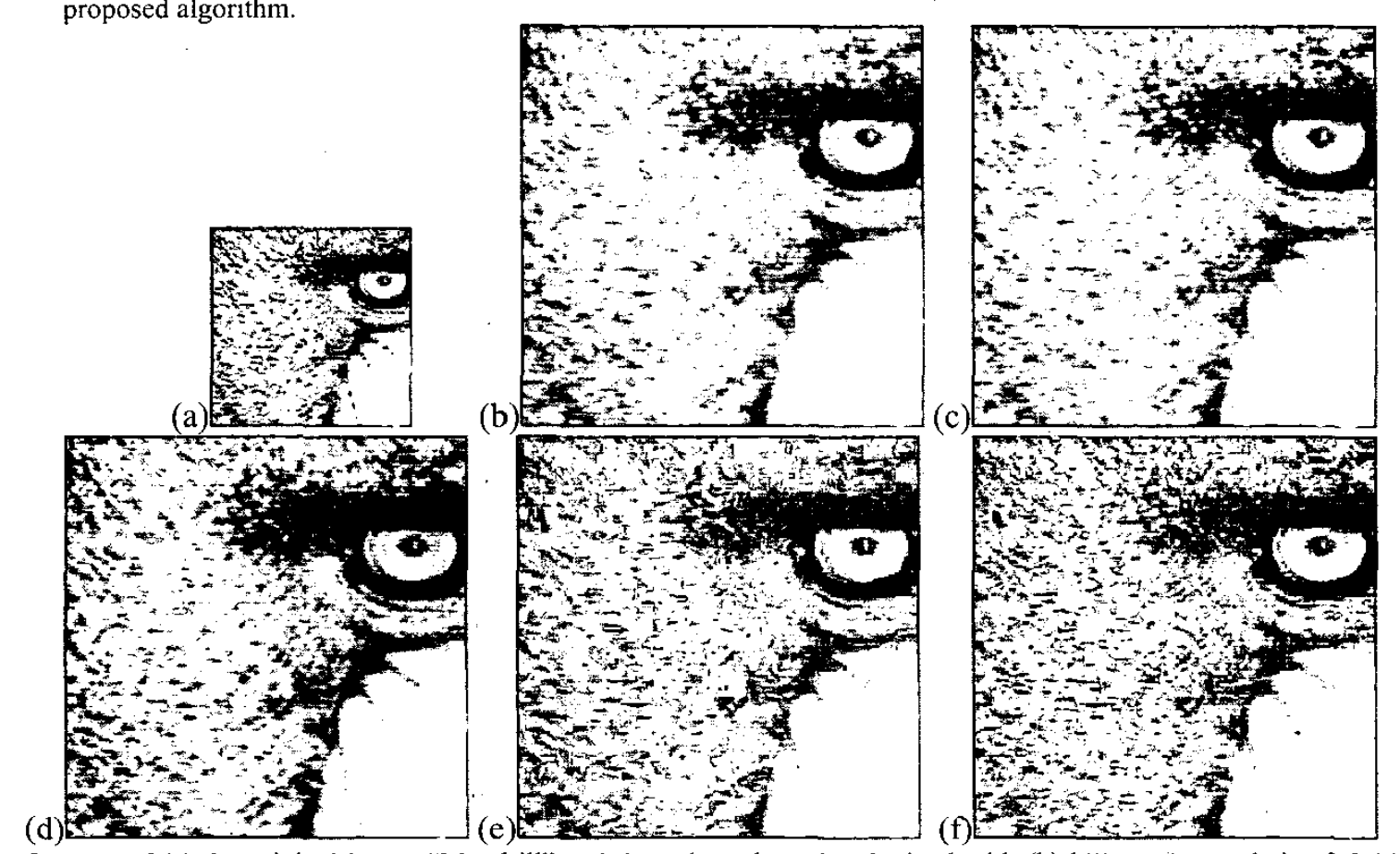

Fig. 2 Parts of (a) the original image "Mandrill" and the enlarged results obtained with (b) bilinear interpolation [1] (c) cubic convolution [3], (d) parametric cubic convolution [5], (e) conventional fractal enlargement [7] and (e) the proposed algorithm. 